\title{
Selection of Optimal Variant of Hybrid System under Conditions of Uncertainty
}

\author{
Matsankov Misho, ${ }^{1,}$, Ivanova Mihaela ${ }^{1}$ \\ ${ }^{1}$ TU-Sofia, EPF-Sliven, Bulgaria
}

\begin{abstract}
The aim of the study is to select an optimal variant of a hybrid system under conditions of uncertainty according to selected criteria. The problems of the study are the different options for building the hybrid system and choosing an adequate criterion for choosing the optimal variant. The study covers analysis of three variants of building up a hybrid system for supplying of autonomous electric load. The three variants are: introduction of an active user for equalizing the load schedule; introduction of additional generating source for covering the peak portion of the load schedule; covering the peak portion of the load by using an additional generating source and diesel generator. An optimal variant is selected under the conditions of uncertainty according to two criteria: minimum discounted expenses and loss of power. To this effect, the method of Mathematical hierarchical game theory analysis is applied. As a result of the study, the appropriate criteria for choosing an optimal variant for building a hybrid system are obtained. The results obtained allow to set a different degree of significance of the formulated basic criteria in the computational algorithm for decision making for the construction of the hybrid system.
\end{abstract}

\section{Introduction}

The choice of option to build a hybrid system is done according to pre-selected criteria of optimality. Creating variants for building a hybrid power supply system for autonomous users takes into account the possibilities of introducing active users, powering decentralized electricity producers based on renewable energy sources (RES) or diesel generators.

Effective use of active users in smart grids allows you to regulate your electricity consumption. The Active User reduces maximum and peak loads in the 24-hour freight schedule, resulting in lower of power losses and losses of energy. The economic effect of switching on the active user is achieved by operating beyond the peak and peak load area [1].

It is possible to supply the autonomous load with decentralized energy sources DES based on (wind power plants, photovoltaic systems, etc.). The feasibility of their construction is proved by carrying out an electric energy audit and a feasibility study.

The building of a hybrid system for power supply of autonomous users working under set up load schedule is performed after the formulation of variants, electric power audit and selection of the optimal one of them according to technical and economic indicators.

The goal of the study is to select the optimal variant of the hybrid system under the conditions of uncertainty according to selected criteria.
To achieve the goal is to select criteria and build options for building a hybrid system and also to estimate the weight coefficients in choosing an optimal variant by applying the hierarchical analysis method of the mathematical theory of games.

\section{Criteria for choosing an optimal solution for building a hybrid system under uncertainty}

The choice of criteria for building a hybrid system is based on capital budgeting methods: net present value; discounted cash flows and an internal rate of return [2,3].

For each option, the discounted cash flows are measured and its net present value is present. This estimate requires the determination of the amount and timing of any additional accumulated cash flows for the option. A negative net present value option is discarded. The option with the highest net present value is accepted.

The internal rate of return is defined as a discount rate that gives a net present value equal to zero. Choose variants that have an internal rate of profit higher than the discount rate.

Theoretically, the methodology for determining net present value is advisable, but it is in practice preferable to calculate the internal rate of return because variants are valued in percentages of return instead of in monetary terms.

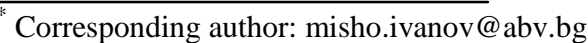


Electricity consumption targets are discounted costs that depend on technical and economic indicators. Power losses are an integral part of the operating costs involved in calculating discounted costs. If a purely technical benchmark is chosen as the criterion for choosing the optimal hybrid system option, the minimum power loss value, which depends on running power, active resistance and rated line voltage, is appropriate. [4]

The expert estimate shows that discounted costs are about a four times more significant criterion than power losses.

The minimum discounted costs and minimum power losses are chosen as criteria for assessing the options for building a hybrid system.

\section{Formulation of variants and justification of selection criteria for hybrid system for supplying of autonomous users}

The variants of building up the hybrid system could be [1]:

- variant A - introduction of an active user for equalizing the load schedule;

- variant B - introduction of an additional generating source to cover the peak portion of the load schedule;

- variant C - covering the peak portion of the load schedule by using an additional generating source and diesel generator.

The formulation of variants for building of a hybrid system for supplying autonomous users takes into consideration the possibilities for introduction of active users, supplying by decentralized generators of electricity, built on the basis of renewable energy sources (RES) or diesel generators [1].

The effective use of active users in Smart Grid allows the adjustment of use of electricity. The active user reduces the maximum and peak loads in the 24-hour load schedule that leads to decreasing losses in power and electricity. The economic effect from including the active user is achieved from its work outside the zone of the maximum and peak load.

The minimization of losses of power by the active users is performed in the following sequence:

- The users of Smart Grid are grouped by types: household, industrial, with automated production and with possibility for switching on and off according to the amount of the power in the load schedule;

- The price of electricity is set according to the tariff zones: night, day and peak ones;

- The 24-hour load schedule of users is constructed;

- An active user that could be included under a set mode is selected.

An inquiry regarding the work of active users was conducted among specialists in the field of power generation. Their expert appraisal is as follows:

- The owner of the electricity user is interested in the uninterrupted power supply at the time they needed such. For them, the benefit from Smart Grid for the active user has no importance.
- The owner of the electricity active is interested in the technological process and its implementation only. Most important for the owner is that once the technological process has started it should be completed without any interruption in the power supply.

- Financial stimulus through executing mutually beneficial contracts between Smart Grid and the owner of the active user only will give a positive result, i.e. the owner of the active user should be convinced to have a contract for payment of the energy used according to low tariff.

- There are technological processes that are impossible to be moved along the load schedule and cannot be selected for active users.

It is possible to supply the autonomous load with decentralized power sources built on the basis of RES (wind plants, photovoltaic systems, small water power plants, etc.). The feasibility of their construction is proved through conducting of power generation audit and technical and economic appraisal.

The selection of a variant for construction of a hybrid system is performed after preliminary chosen criteria of optimality.

\section{Selection criteria of optimal solution regarding construction of hybrid system after uncertainty conditions}

A suitable technical and economic criterion for power generation sites is the discounted expenses $R_{D}$, that are expressed by the following equation [2]:

$$
R_{D}=\sum_{t=1}^{T_{c}} \sum_{i=1}^{n} K_{i t} a^{t-1}+\sum_{t=1}^{T_{c}} \sum_{i=1}^{n} \sum_{j=1}^{m} C_{i j t} a^{t-1}
$$

where $K_{i t}$ are the investments in the $i$-element of the scheme in year $t ; C_{i j t}$ - the operational costs in the $i^{t h}$ element of the scheme of $\mathrm{j}^{\text {th }}$ kind for year $t ; T_{\mathrm{c}}$ operational period; $n$ - the number of elements of the scheme; $m$ - the number of kinds of operational costs; $\alpha-$ the ratio of discounting which depends on the duration of the period of calculation and the discounting rate.

The operational costs $C_{i j t}$ in $i^{\text {th }}$ elements consist of components with the respective indices: $j=1$ maintenance and repair; $\mathrm{j}=2$ - reconstruction and modernization; $j=3$ - losses of active power in the element [2]:

$$
C_{i j t}=\sum_{j=1}^{3} C_{i t}=p_{1} K_{i t}+p_{2} K_{i t}+\left(\Delta P_{\text {max }} \tau \beta\right)_{i t}
$$

where $\mathrm{p}_{1}$ and $\mathrm{p}_{2}$ are the rated values of the operational costs, for maintenance and reconstruction of power generation, respectively; $\Delta P_{\max }$ - the losses of active power in a mode of maximum load; $\tau$ - the fictious duration of maximum losses of power; $\beta$ - the price of electricity.

The losses of power are component of the operational costs and participate in the calculation of the discounted expenses. Thus the discounted expenses could be discussed as technical and economic indicator. 
If a purely technical indicator is selected as selection criterion of optimal variant of hybrid system, the minimum value of power loss is suitable.

The expert assessment of the relative weights of the three options according to the two criteria shall take into account the following:

- The maximum effect for option 1 will be obtained if the active user is switched on from 22 to 6 hours and switches off from $8 \mathrm{am}$ to $1 \mathrm{pm}$ and from $17 \mathrm{~h} 00$ to $21 \mathrm{~h}$. Then the load schedule is almost aligned - with a $25 \%$ reduction in load peak power time.

- In the evaluation of the options, besides the investments and the operating costs, as well as the value of the control facilities and the means of automatic switching on or off of the active user respectively; the additional generating source and the diesel generator.

The results of the expert assessment for the relative weights of options 1, 2 and 3 are respectively:

- by criterion loss of power: $1 ; 1,2$ and 1,3;

- by the Discounted Cost criterion: $1 ; 1.5$ and 1.7.

Optional studies and their comparison with the selected criteria aim to achieve minimal technical and economic indicators.

The resulting relative weights of variants 1,2 and 3 are used in applying the mathematical theory of games to select the optimal variation.

\section{Application of mathematical game theory for selection of optimal variant for building of hybrid system}

One of the approaches for accepting decisions under the uncertainty conditions is based on the application of the hierarchical analysis method [5], [6], [7].

In order to select the best variant, two main criteria are formulated: loss of power and discounted expenses. According to the conducted expert appraisal, the discounted expenses are about 4 times more significant as criterion compared to the losses of power, since they represent part of the operational costs that are one of the components of the discounted costs [1].

A weight ratio $\mathrm{p}_{1} \%$ is accepted for the discounted expenses, and $\mathrm{P}_{2} \%$ for the loss of power. The analysis for appraisal of the three variants is conducted from the point of view of the losses of power and the discounted expenses (Table 1).

Table 1. Variants and Criteria for Construction of Hybrid System

\begin{tabular}{|l|c|c|c|}
\hline & \multicolumn{3}{|c|}{ Variant } \\
\hline Criteria & $\mathrm{A}$ & $\mathrm{B}$ & $\mathrm{C}$ \\
\hline Loss of power & $\mathrm{p}_{11}$ & $\mathrm{p}_{21}$ & $\mathrm{p}_{31}$ \\
\hline $\begin{array}{l}\text { Discounted } \\
\text { expenses }\end{array}$ & $\mathrm{p}_{12}$ & $\mathrm{p}_{22}$ & $\mathrm{p}_{32}$ \\
\hline
\end{tabular}

The structure of the decision-making problem is pointed out at Fig. 1. The problem is solved in respect to two criteria (loss of power and discounted expenses) and three variants (A, B and $\mathrm{C}$ ).

Figure 1 shows the structure of weight factor formation in the selection of the hybrid system.

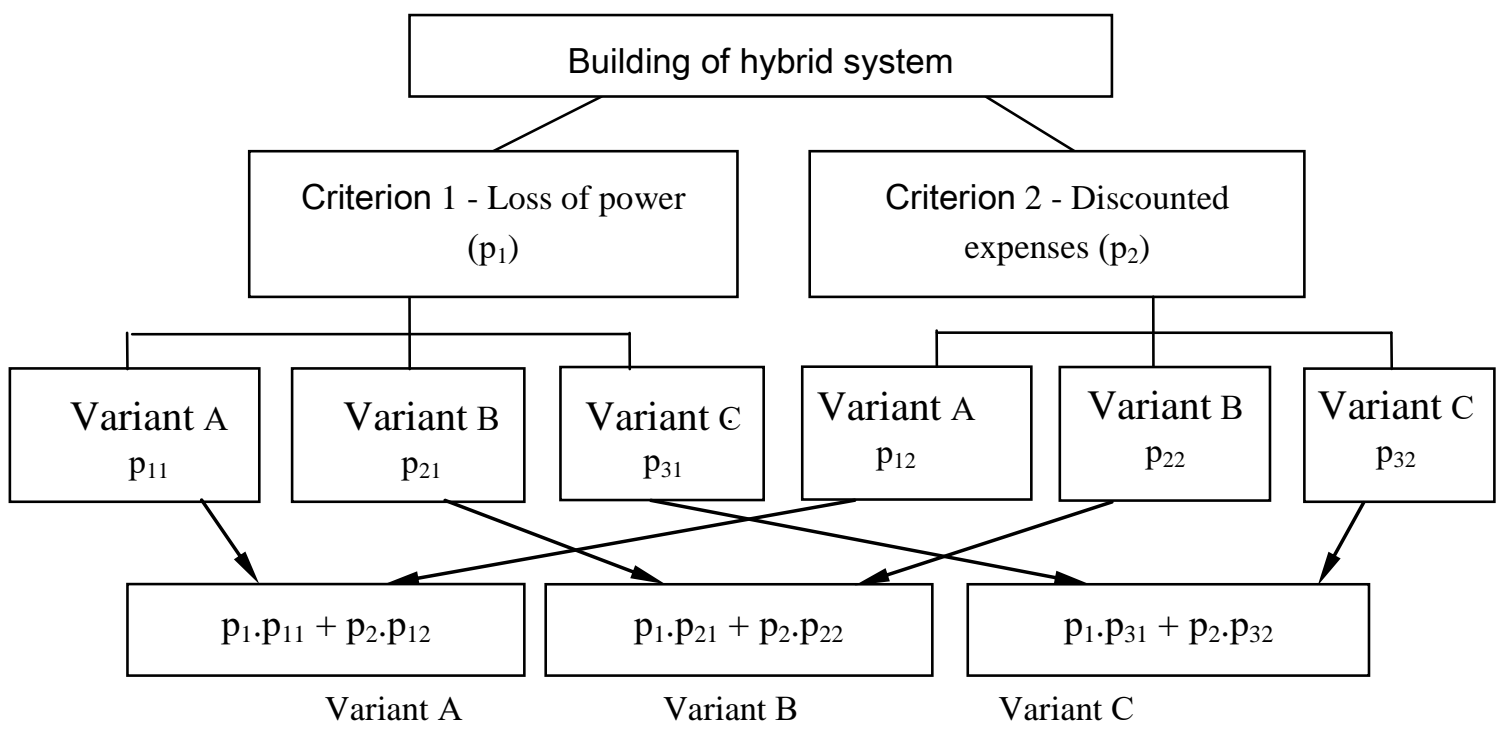

Fig.1. Structure of problem for variant selection regarding building of hybrid system

The discounted expenses are about 4 times more significant as criterion compared to the loss of power. The mathematical apparatus of the theory of games is applied [8,9].

The value of 4 was set for the element $(2,1)$ of matrix A, then $a_{21}=4$, and element $a_{12}=1 / 4$. The criteria $R$ and $L$ for the discounted expenses and the losses of power, respectively, and the matrix of comparison are expressed by: 


$$
A_{R}=\begin{array}{cc}
L & R \\
R & L\left(\begin{array}{cc}
1 & 1 / 4 \\
4 & 1
\end{array}\right) .
\end{array}
$$

The following is received for the normalized matrix $\mathrm{N}$ :

$$
L \quad R
$$

$$
N={ }_{R}^{L}\left(\begin{array}{ll}
0,2 & 0,2 \\
0,8 & 0,8
\end{array}\right) .
$$

The average values of the row elements are $w_{R}=0,8$ and $w_{L}=0,2$ i.e. the weights that are shown in fig. 2 .

The relative weights of the variant solutions A, B and $\mathrm{C}$ are calculated within the limits of each criterion $\mathrm{R}$ and $L$ by using both comparison matrices:

$$
\text { A } \quad B \quad C
$$

$$
A_{L}=\begin{array}{r}
A \\
C
\end{array}\left(\begin{array}{ccc}
1 & 0,83 & 0,77 \\
1,2 & 1 & 0,83 \\
1,3 & 1,2 & 1
\end{array}\right) .
$$

The sum of the elements of the piles $[3,5 ; 3,03 ; 2,6]$.

$$
\begin{array}{lll}
A & B & C
\end{array}
$$

$$
A_{R}=B\left(\begin{array}{ccc}
1 & 1,5 & 1,7 \\
0,67 & 1 & 1,13 \\
0,58 & 0,88 & 1
\end{array}\right) .
$$

The sum of the elements of the piles

$[2,25 ; 3,38 ;$ $3,83]$.

The elements of the matrices $\mathbf{A}_{R}$ and $\mathbf{A}_{L}$ are determined on the basis of the preliminary conducted energy audit, concerning the relative humidity of the three variants $A, B$ and $C$.
A $\quad B \quad C$

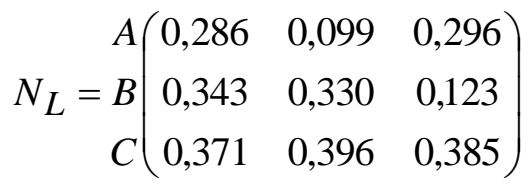

with the average value of the row elements:

$$
\begin{gathered}
w_{L A}=(0,286+0,099+0,296) / 3=0,23 ; \\
w_{L B}=(0,343+0,33+0,123) / 3=0,265 ; \\
w_{L C}=(0,371+0,396+0,385) / 3=0,384
\end{gathered}
$$

and

$$
\begin{aligned}
& A \quad B \quad C
\end{aligned}
$$

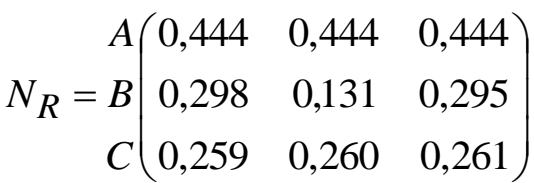

with the average value of the row elements:

$$
\begin{aligned}
& w_{R A}=(0,444+0,444+0,444) / 3=0,444 ; \\
& w_{R B}=(0,298+0,131+0,295) / 3=0,241 ; \\
& w_{R C}=(0,259+0,26+0,261) / 3=0,26 .
\end{aligned}
$$

The values $\left(w_{R A}, w_{R B}, w_{R C}\right)=(0,444 ; 0,241 ; 0,26)$ give the respective weights for the variants $\mathrm{A}, \mathrm{B}$ and $\mathrm{C}$ according to the second criterion - discounted expenses. The values $\left(w_{L A}, w_{L B}, w_{L C}\right)=(0,23,0,265,0,384)$ are the relative weights referring to the first criterion. - loss of power.

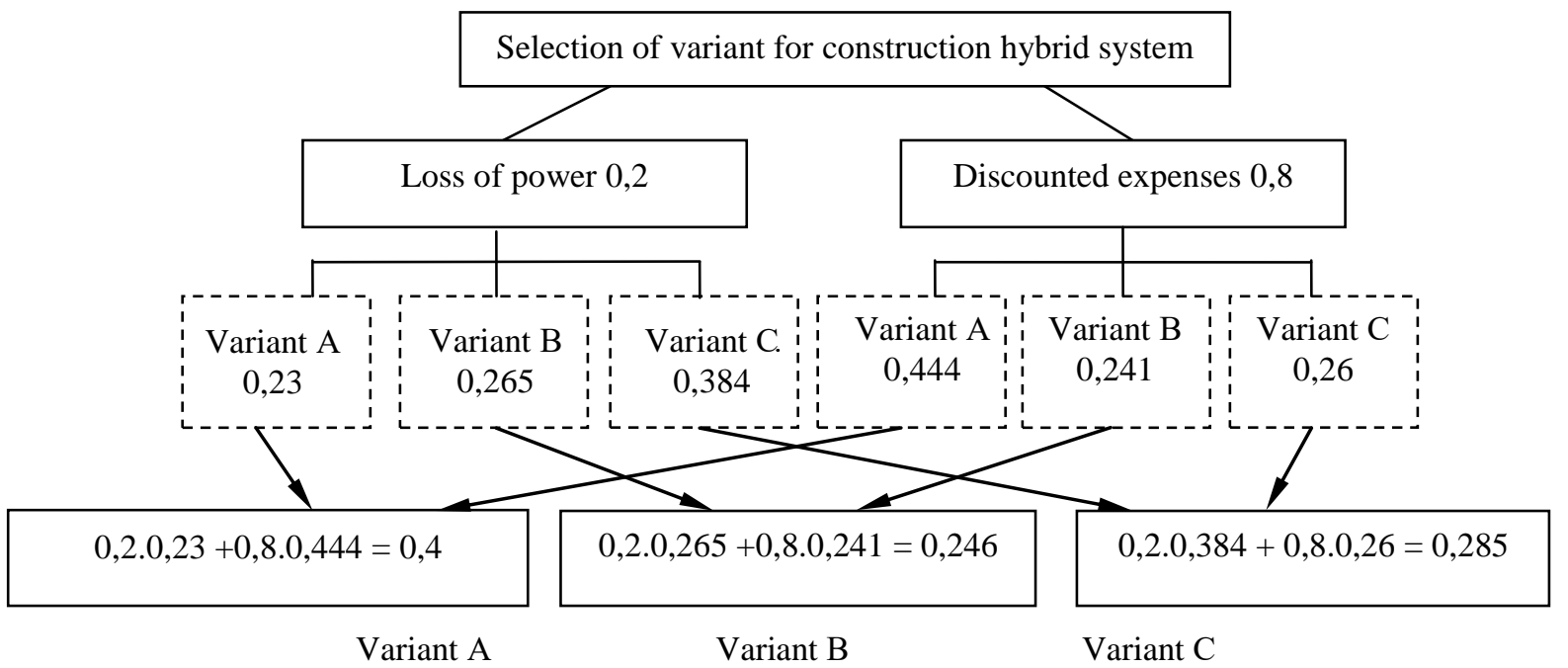

Fig. 2. Selection of Optimal Variant of Hybrid System

As a result of the calculations, the weight coefficients in the structure of the choice of option for the construction of the hybrid system, which are shown in fig. 2 .

A relative weight ratio of $80 \%$ is received for the discounted expenses, and for the loss of power - $20 \%$. The appraisal results of the three variants in respect to loss of power and discounted expenses are given in Table 2 .
Table 2. Relative Weight Ratios for Criteria Regarding Decision-Making for Building of Hybrid System

\begin{tabular}{|l|c|c|c|}
\hline & \multicolumn{3}{|c|}{ Variants } \\
\hline Criteria & $\mathrm{A}$ & $\mathrm{B}$ & $\mathrm{C}$ \\
\hline Loss of power & $23 \%$ & $26,5 \%$ & $38,4 \%$ \\
\hline $\begin{array}{l}\text { Discounted } \\
\text { expenses }\end{array}$ & $44,4 \%$ & $24,1 \%$ & $26 \%$ \\
\hline
\end{tabular}


The structure of the decision-making problem with the calculated ratios is shown in Fig. 2.

\section{Determining of combined weight ratios for variants}

The appraisal of the three variants is based on calculation of the combined weight ratios for each of them.

- Variant A: 0,2.0,23+0,8.0,444=0,4;

- Variant B: 0,2.0,265+0,8.0,241 = 0,246;

- Variant C: 0,2.0,384 + 0,8.0,26=0,285.

Based on these calculations, variant A receives the higher combined weight and represents the optimal selection.

\section{Conclusions}

- The calculation algorithm allows setting up of different degree of significance of the formulated basic criteria for decision-making in respect to buildings of the hybrid system.

- The discounted expenses of the hybrid system are much more significant criterion than the loss of power.

- The comparison of the results from the calculations shows that for each of the reviewed cases variant $A$ is the optimal one in respect to both criteria: discounted expenses and loss of power.

\section{Acknowledgements}

The autors would like to thank the Research and Development Sector at the Technical University of Sofia for financial support.

\section{References}

1. Matsankov M., M. Ivanova. Energy audit to build a hybrid system. ISSN 1312-3920, Notices of TUSliven, No 1, 2019

2. Bakardjieva J. N. Optimal configuration and seconding in distribution networks with decentralized sources. Thesis of HMTU, Sofia, 2014.

3. Andonov A, J. Bakardjieva Criteria for choosing an optimal configuration and a node to segment the branches in a medium voltage distribution network., Energetics magazine No 6,2012, pp. 52 - 57

4. Nedelcheva S I, Electrical networks, TU - Sofia Publishing house, Sofia, 2005.

5. Nedelcheva S.I., M.Matsankov, I.Lazarov. Application of Probability Theory and Mathematical Statistics in Electric Power Engineering, ISSN 13123920, Notices of TU-Sliven, No 6, 2018.

6. Nedelcheva S., I. Lazarov. Selection of an optimal option for building wind farms using the hierarchical analysis method. "Notices of TU-Sliven", ISSN 13123920, No 6, 2015, p.7-12.

7. Lazarov I. J. Optimal modes of operation of decentralized energy sources under conditions of uncertainty. Thesis on TU-Sofia, 2017.

8. Hazewinkel M. Games theory. Springer Science+Business Media B.V. Kluwer Academic Publishers, ISBN 978-1-55608-010-4, 2001.

9. Elmer G. Wiens. Game Theory. http://www.egwald.ca/operationsresearch/gameintrod uction.php 